\title{
ESTUDO DA DISSOLUÇÃO DE ADIÇÕES CONTENDO MGO EM ESCÓRIAS DE ACIARIA EM SEU IMPACTO NA FORMAÇÃO DE MAGNESIO-WUSTITA*
}

\author{
Alan Alves Vieira ${ }^{1}$ \\ Antônio Augusto Martins ${ }^{2}$ \\ Rafaela Pereira Batista ${ }^{3}$ \\ Fabiane Roberta Freitas da Silva ${ }^{4}$ \\ Roberto Ribeiro de Avillez ${ }^{5}$ \\ André Luiz Vasconcellos da Costa e Silva ${ }^{6}$
}

\begin{abstract}
Resumo
Uma das maneiras mais eficientes de aumentar a campanha dos refratários de conversores é o uso de técnicas de slag splashing e slag coating. Nestes processos, antes de sua execução, a escória de fim de sopro é modificada pela adição de materiais contendo, principalmente, $\mathrm{MgO}$. Escórias de conversor contendo alto teor de $\mathrm{FeO}$ tem, como fase de equilíbrio mais provável a magnesiowustita (MW). Este estudo visa determinar se, nos curtos tempos de processo em conversor, e sempre a temperaturas bastante inferiores ao ponto de fusão do $\mathrm{MgO}$, ocorre a dissolução do $\mathrm{MgO}$ e a saturação da escória em MW ou se a escória se mantém fora de equilíbrio. Os resultados indicam que a MW é formada e, em muitos casos, sua composição pode ser prevista com razoável precisão através de cálculos de equilíbrio por termodinâmica computacional.
\end{abstract}

Palavras-chave: Conversor LD; Refratário; Tratamentos; Escória; Magnésio-Wustita.

\section{EVALUATION OF THE DISSOLUTION OF MGO CONTAINING ADDITIONS IN STEELMAKING SLAG AND ITS IMPACT ON THE FORMATION OF MAGNESIOWUSTITE}

\section{Abstract}

One of the most efficient ways to increase the life of converter refractories is the use of slag splashing and slag coating techniques. In these techniques the end-of-blow slag is usually modified by the addition of materials containing, mainly, MgO. Basic converter slags containing high $\mathrm{FeO}$ have, as the most probable equilibrium phase, magnesiowustite (MW). This study aims at determining if, in the short process times in the converter, at temperatures well below the melting point of $\mathrm{MgO}, \mathrm{MgO}$ dissolution occurs and the slag saturates in $\mathrm{MW}$ or if the slag remains out of equilibrium with respect to $\mathrm{MgO}$ rich phases. The results indicate that $\mathrm{MW}$ is formed and, in many cases, its composition can be predicted with reasonable precision by computational thermodynamics calculations.

Keywords: LD converter; Refractory; Treatments; Slag; Magnesium-Wustite.

1 Aluno de Engenharia Metalúrgica, Técnico de Desenvolvimento, Aciaria, EEIMVR-UFF / Companhia Siderúrgica Nacional, Volta Redonda, RJ, Brasil

2 Engenheiro Metalúrgico, Coordenador de Projetos Especiais, Aciaria, Companhia Siderúrgica Nacional, Volta Redonda, RJ, Brasil

3 Engenheira Metalúrgica, Engenheira de Desenvolvimento, Aciaria, Companhia Siderúrgica Nacional, Volta Redonda, RJ, Brasil

4 Engenheira Metalúrgica, D.Sc., Professora Adjunta, EEIMVR-UFF, Volta Redonda, RJ, Brasil.

5 Engenheiro Metalúrgico, PhD, Professor, PUC-RIO, Rio de Janeiro, RJ, Brasil.

6 Engenheiro Metalúrgico, PhD, Professor, EEIMVR-UFF, Volta Redonda, RJ, Brasil. 


\section{INTRODUÇÃO}

Conversores a oxigênio necessitam longas campanhas para garantir sua economicidade [1] em função dos elevados custos dos revestimentos refratários e das paradas associadas ao processo de revestimento [2]. Uma das maneiras eficientes de aumentar a campanha dos refratários de conversores é o uso de técnicas de slag splashing e slag coating. Nestes processos, usualmente, a escória de fim de sopro é modificada pela adição de materiais contendo, principalmente, $\mathrm{MgO}$ antes de sua reação com os refratários. Embora seja comum na operação de fornos elétricos e conversores discutir-se a saturação em $\mathrm{MgO}$, escórias contendo alto teor de $\mathrm{FeO}$ e adições de $\mathrm{MgO}$ tem, como fase de equilíbrio mais provável a magnesiowustita (MW) [3] pois $\mathrm{FeO}, \mathrm{MgO}$ e $\mathrm{CaO}$ tem a mesma estrutura cristalina (halita) e o sistema MgOFeO apresenta miscibilidade completa no estado sólido, sendo a fase sólida formada a chamada MW. A Figura 1 apresenta os limites de saturação no sistema e a Figura 2 o sistema isomorfo FeO-MgO. A existência de duas valências do ferro sempre é um complicador adicional nestes sistemas. Como parte do desenvolvimento do processo de slag splashing e slag coating, este estudo visa determinar se, nos curtos tempos de processo em conversor, e a temperaturas sempre bastante inferiores ao ponto de fusão do $\mathrm{MgO}$, ocorre a dissolução do $\mathrm{MgO}$ e a saturação da escória em MW ou se a escória se mantém fora de equilíbrio. Evidentemente, há um razoável número de trabalhos sobre a cinética de dissolução de cales e carbonatos (dolomita, etc) em escórias p.ex. refs. [4-7]. Entretanto, quase a totalidade destes trabalhos é realizado em condições muito distantes daquelas reinantes em um conversor ou em um forno elétrico a arco.

Amostrando-se escórias formadas durante o processo de conversão, é possível verificar se, efetivamente, as fontes de $\mathrm{MgO}$ utilizadas dissolvem-se nas escórias e se ocorre a precipitação de compostos como a magnesiowustita. Esta verificação é de grande importância para o projeto do processo de slag splashing e slag coating. Adicionalmente, como a termodinâmica computacional representa uma ferramenta de grande importância no projeto do processo de splashing e coating, estas amostragens podem ser úteis para a comparação com cálculos termodinâmicos, indicando acertos, desvios e dificuldades com os bancos de dados disponíveis [8]

Assim, os objetivos deste trabalho são: através da amostragem de escórias reais de final de sopro e a serem empregadas para slag splashing ou slag coating, avaliar a extensão da dissolução das fases ricas em MgO adicionadas e a eventual formação de soluções sólidas como a magnesiowustita. Comparar os resultados obtidos na caracterização experimental com resultados calculados por termodinâmica computacional empregando dois diferentes bancos de dados usuais para o estudo de escórias. 


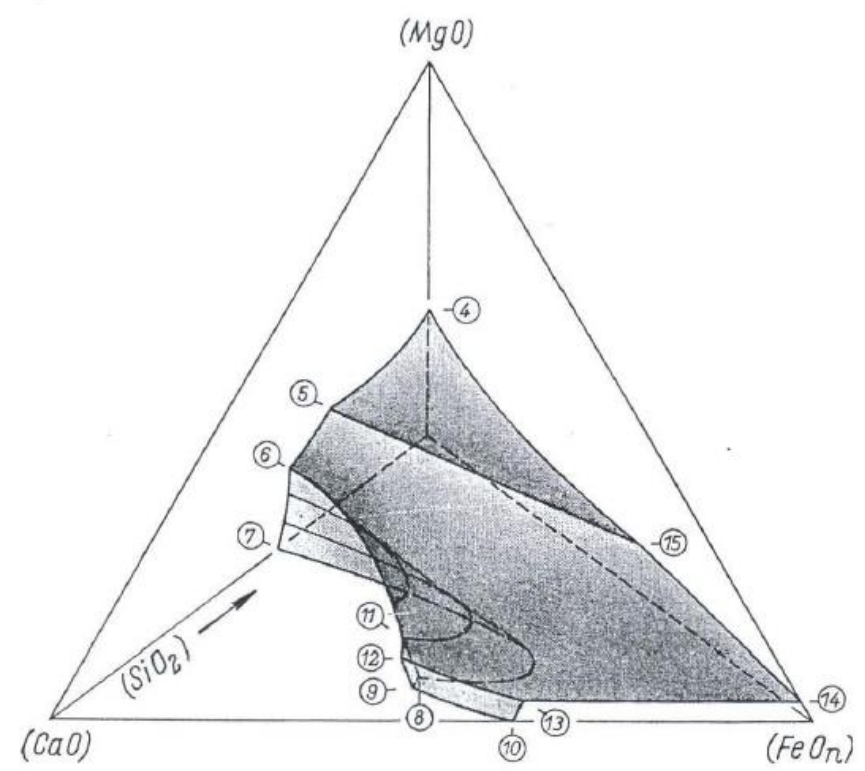

Figura 1. Representação esquemática tridimensional do diagrama de equilíbrio de fases $\mathrm{CaO}-\mathrm{FeOn}$ $\mathrm{SiO} 2-\mathrm{MgO}$ indicando as superfícies de saturação: Saturação em halita ou magnesiowustita ( $\mathrm{Fe}, \mathrm{Ca}, \mathrm{Mg}) \mathrm{O}$, superfície: 12-13-14-15-5-6--11-12. Saturação em olivina 2(Mg, $\mathrm{Fe}, \mathrm{Ca}) \mathrm{O} . \mathrm{SiO}_{2}$, superfície 5-15-4-5. Adaptado de [3]

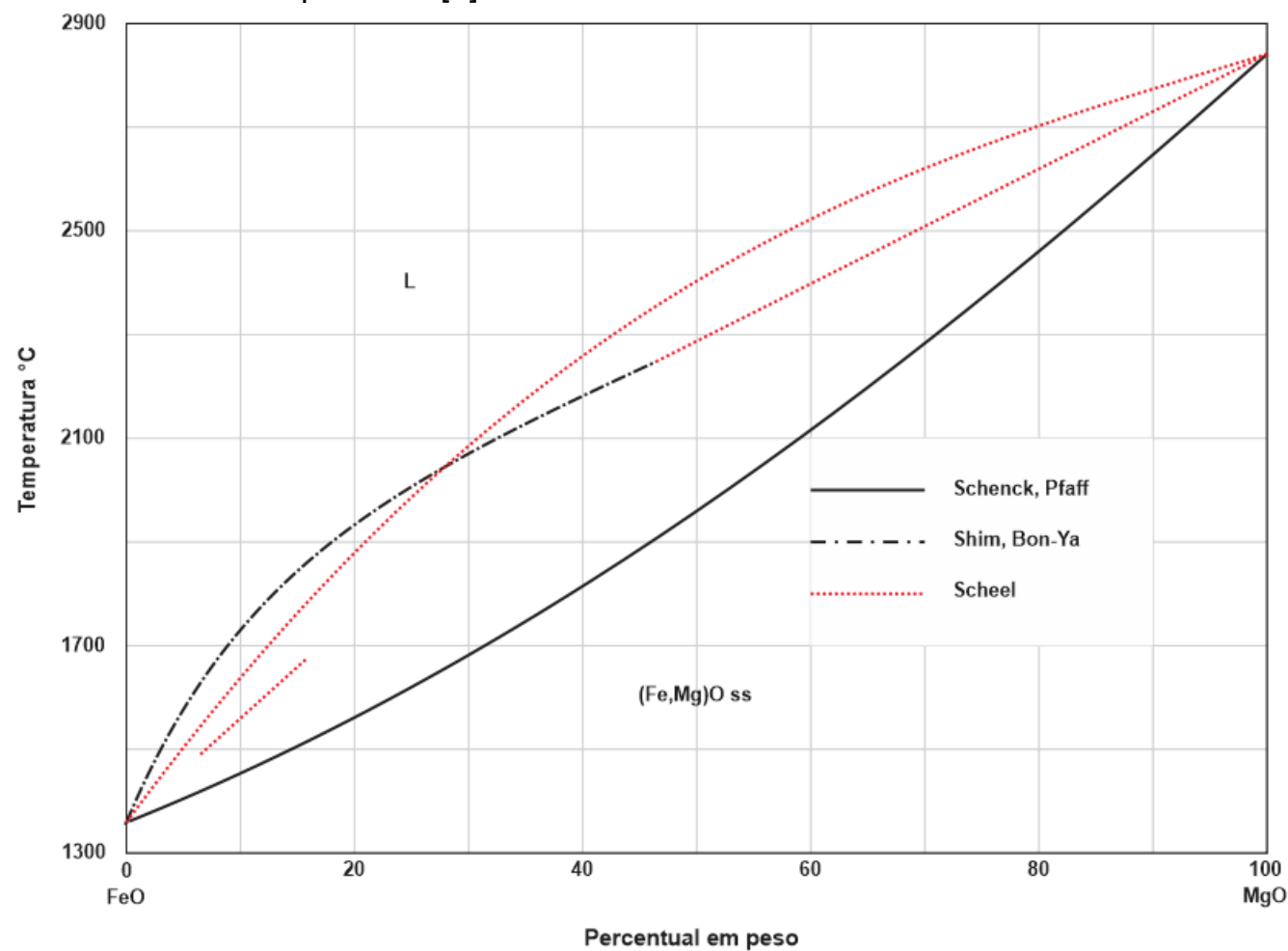

Figura 2. Diagrama de equilíbrio de fases experimental FeO-MgO. Adaptado de [9]

\section{MATERIAIS E MÉTODOS}

Foram amostradas escórias de 16 corridas ao final do sopro em um conversor de 230t. Dentre estas, 16 escórias retidas no conversor para a realização de slag splashing ou coating também foram amostradas. Todas as corridas das quais as escórias foram amostradas foram de aço semelhante ao AISI 1006 e transcorreram sem anormalidades (ressopro, adição de refrigerante, interrupções, etc.). As amostras de escória foram analisadas por quatro técnicas: 
a) Análise química por espectrometria de raios-X em escórias moídas e compactadas em pastilhas, no laboratório da aciaria de planos da CSN;

b) Análise por difração de raios-X com ajuste de parâmetros de rede através do método de Rietveld, para a determinação da fração de fases presentes e, no caso de soluções sólidas, de suas composições aproximadas.

c) Análise por petrografia ótica em amostras montadas, sob vácuo, em resina de poliéster, e polidas.

d) Análise por MEV e EDS de amostras selecionadas entre as amostras do item c), acima.

\subsection{Cálculos termodinâmicos}

Para os cálculos termodinâmicos foi empregado o programa Thermo-Calc versão 2016b, o banco de dados TCOX6 e uma modificação do banco de dados SLAG3 descrita a seguir. Como o banco de dados SLAG3 não contém a descrição termodinâmica da fase magnesiowustita, esta fase foi introduzida, empregando como propriedades dos "end-members" ( $\mathrm{FeO}$ e $\mathrm{MgO}$ ) aquelas já descritas neste banco de dados e ajustando a descrição da fase sólida aos dados da literatura [9] como mostra a Figura 2. Não foi feito nenhum ajuste a descrição termodinâmica da fase líquida, em vista da possibilidade de alterar a descrição de forma incorreta nos demais sistemas contidos neste banco de dados.

A Figura 3 mostra a comparação dos resultados calculados por esta descrição termodinâmica com os dados extraídos da Figura 2.

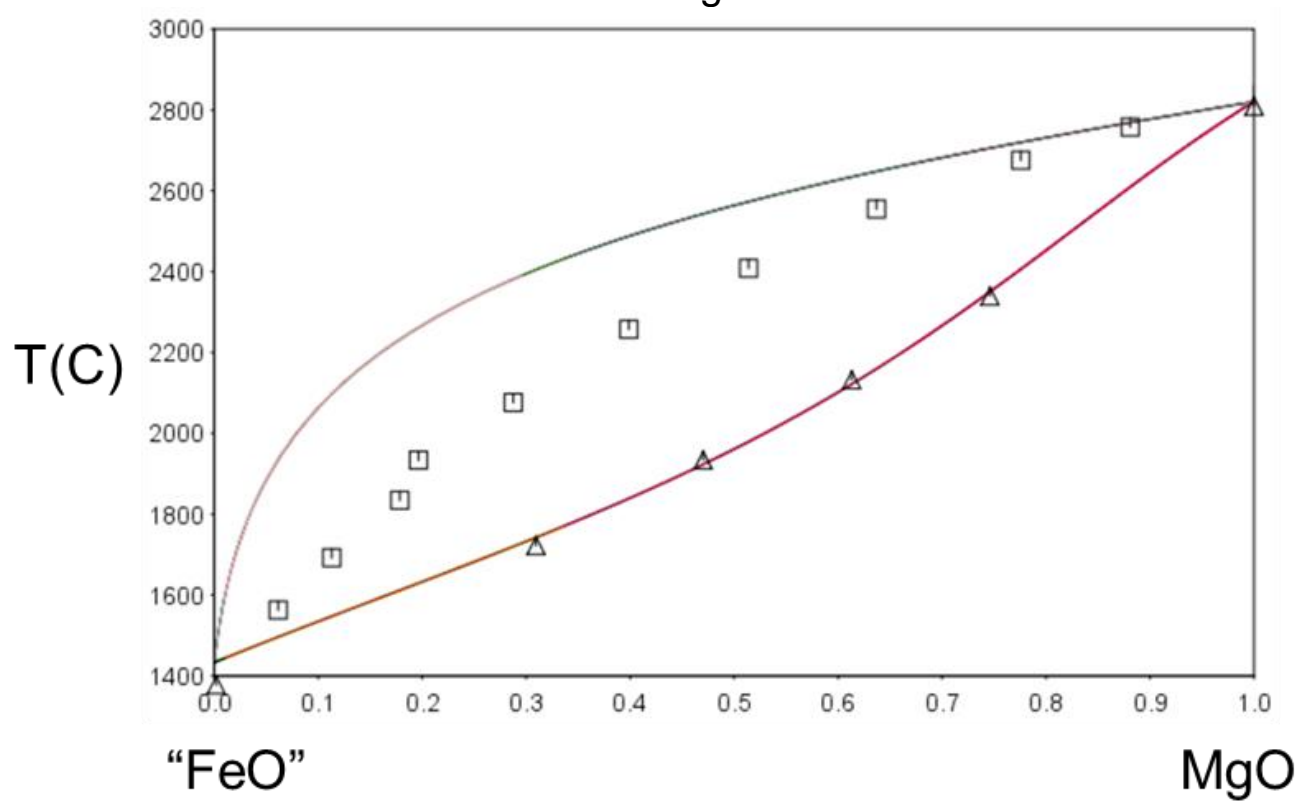

Figura 3. Diagrama pseudo-binário FeO-MgO obtido com a descrição termodinâmica da fase magnesiowustita ( $\mathrm{Fe}, \mathrm{Mg}) \mathrm{O}$ no banco de dados SLAG3. 
A Figura 4 demonstra, esquematicamente, os momentos de amostragem da escória.

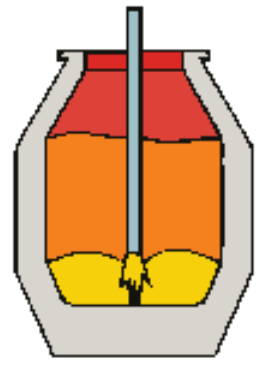

(a)

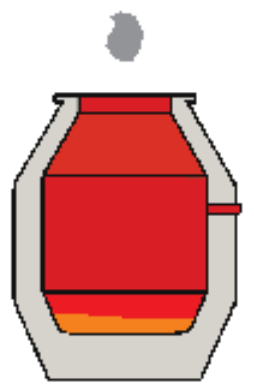

(d)

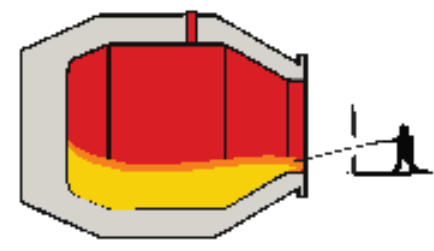

(b)

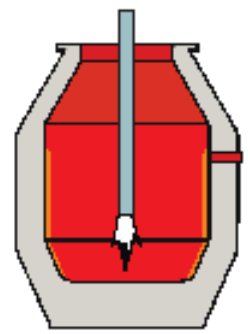

(e)

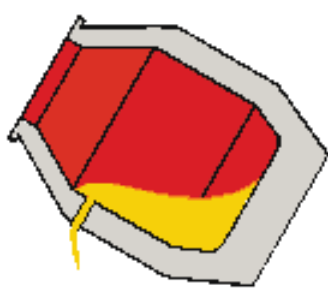

(c)

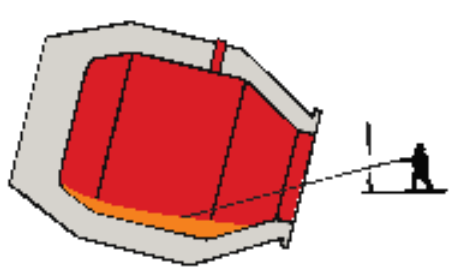

(f)

Figura 4. Representação das etapas de amostragem de escória no processo de conversão. (a) Processo de refino do aço e formação da escória; (b) Amostragem da escória de fim de sopro; (c) Vazamento da corrida em panela; (d) Adição de cales para otimizar qualidade da escória; (e) Processo de slag splashing; (f) Amostragem da escória de final de tratamento. $\mathrm{Na}$ etapa (e) outro processo alternativo seria o slag coating.

\section{RESULTADOS E DISCUSSÃO}

Os resultados das análises realizadas em acordo com o descrito no item dois são descritos para algumas escórias representativas, selecionadas entre as várias amostradas.

\subsection{Resultados da Corrida "214" \\ 3.1.1 Escória após o fim de sopro}

As Figuras 5 e 6 apresentam observações no MEV da amostra de escória após fim de sopro da corrida "214". 

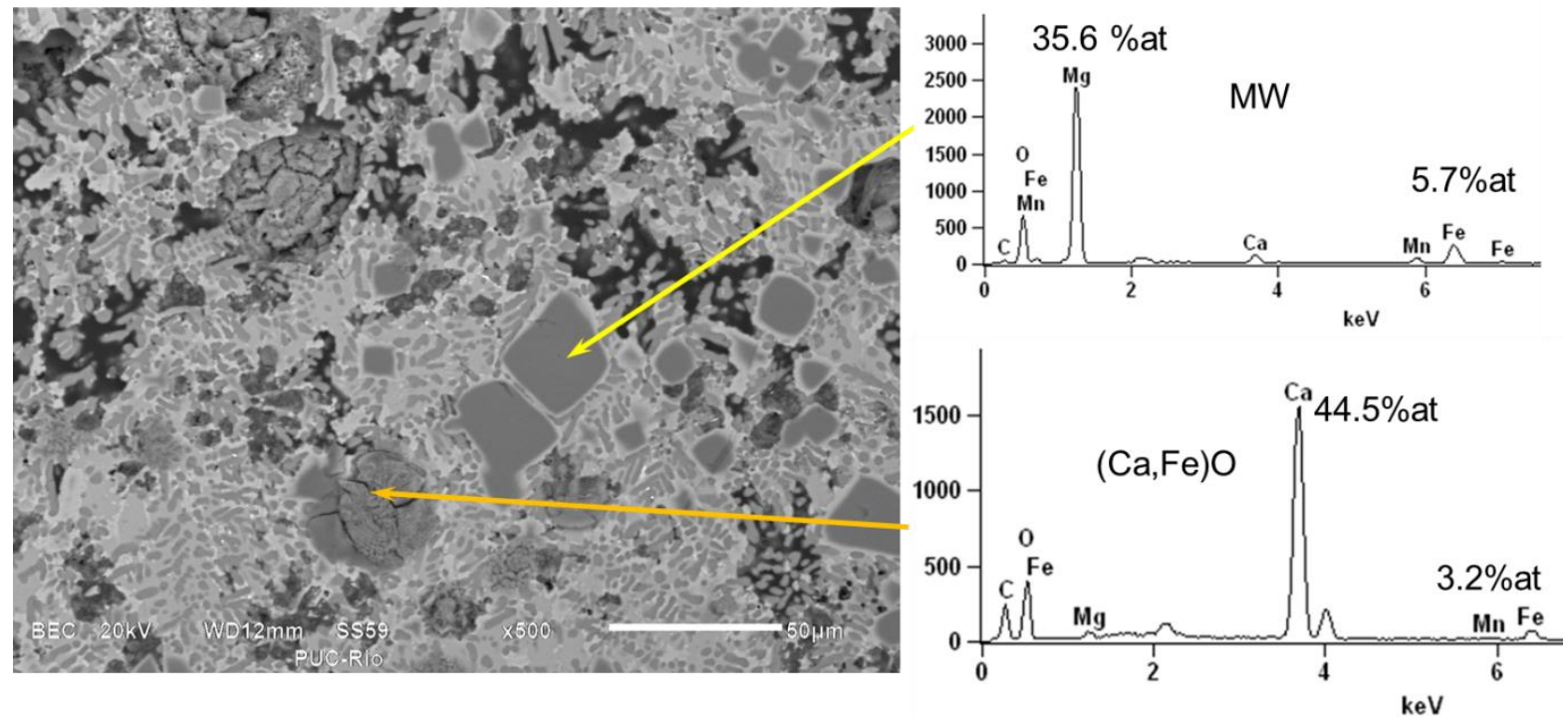

Figura 5. Imagem de elétrons secundários (SE) no MEV de amostra da escória após o fim de sopro da corrida "214". Uma das partículas de magnesiowustita, (com formato aproximado regular) é indicada assim como a análise por EDS deste ponto. Uma partícula de solução sólida $(\mathrm{Ca}, \mathrm{Fe}) \mathrm{O}$ e sua análise por EDS são também indicados.

A morfologia e tamanho das partículas de MW observadas na Figura 5 parecem indicar que esta fase estava presente no estado sólido, na escória. Da mesma forma, observa-se partículas grandes de solução sólida $(\mathrm{Ca}, \mathrm{Fe}) \mathrm{O}$ que também parecem estar presentes, no estado sólido, na escória.

A avaliação microscópica parece indicar que a escória está saturada em (Mg,Fe)O e $(\mathrm{Ca}, \mathrm{Fe}) \mathrm{O}$. Os resultados dos cálculos termodinâmicos são comparados com estas observações na Tabela 1.

Tabela 1. Fases em que a escória de fim de sopro da corrida "214" estaria saturada

\begin{tabular}{|c|c|c|c|}
\hline Método & MEV+EDS & TCOX & SLAG3 mod. \\
\hline Fases & $(\mathrm{Ca}, \mathrm{Fe}) \mathrm{O}$ e MW & $(\mathrm{Ca}, \mathrm{Fe}) \mathrm{O}$ e MW & MW \\
\hline
\end{tabular}

É importante notar que o banco de dados SLAG3 modificado não foi alterado para incluir soluções sólidas $(\mathrm{Ca}, \mathrm{Fe}) \mathrm{O}$, de modo que seria impossível prever a presença desta fase com este banco de dados.

O resultado da análise de EDS da partícula de MW foi comparado com os valores de $\% \mathrm{FeO}$ e \%MgO obtidos pelo exame por DRX e calculados no equilíbrio, na temperatura de fim de sopro com a composição química média da escória, usando os dois bancos de dados citados acima. O resultado da comparação é mostrado na Tabela 2.

Tabela 2. Teor de \%MgO medido e calculado na MW da escória de fim de sopro da corrida "214"

\begin{tabular}{|c|c|c|c|c|}
\hline Método & DRX Rietveld & EDS & TCOX & SLAG mod. \\
\hline$\% \mathrm{MgO}$ & 77 & 78 & 74 & 71 \\
\hline
\end{tabular}

A Figura 6 apresenta outra característica importante observada nas escórias contendo MW: A presença de uma região rica em $\mathrm{FeO}$ em torno das partículas de MW. 

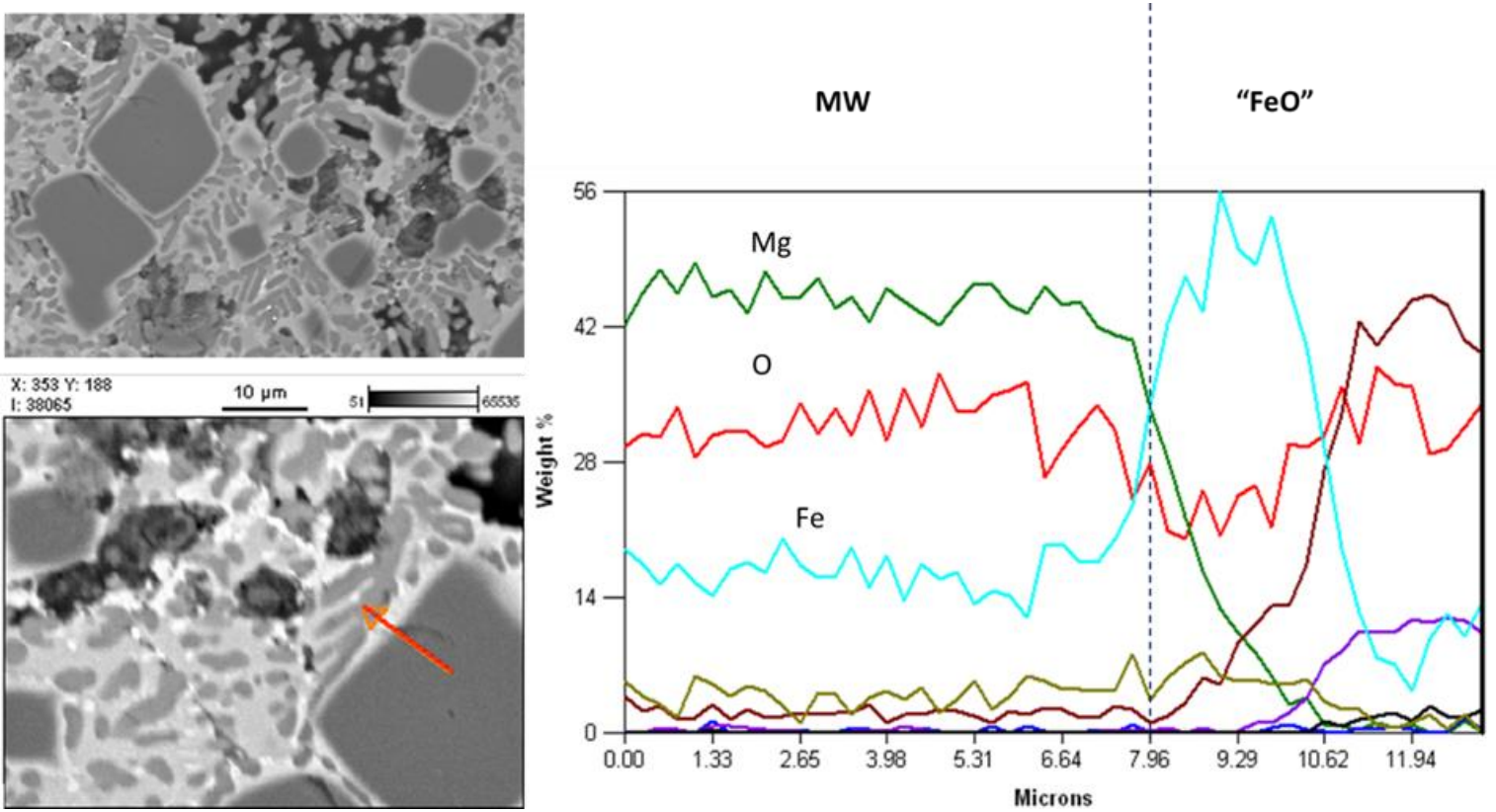

Figura 6. Escória após final de sopro da corrida "214". Análise por EDS em linha, ao longo da linha vermelha indicada na foto obtida por MEV. A formação de uma camada rica em FeO entorno das partículas de geometria regular de MW é evidente.

\subsubsection{Escória após o tratamento}

As Figuras 7 e 8 apresentam observações no MEV da amostra de escória após o tratamento da corrida "214".
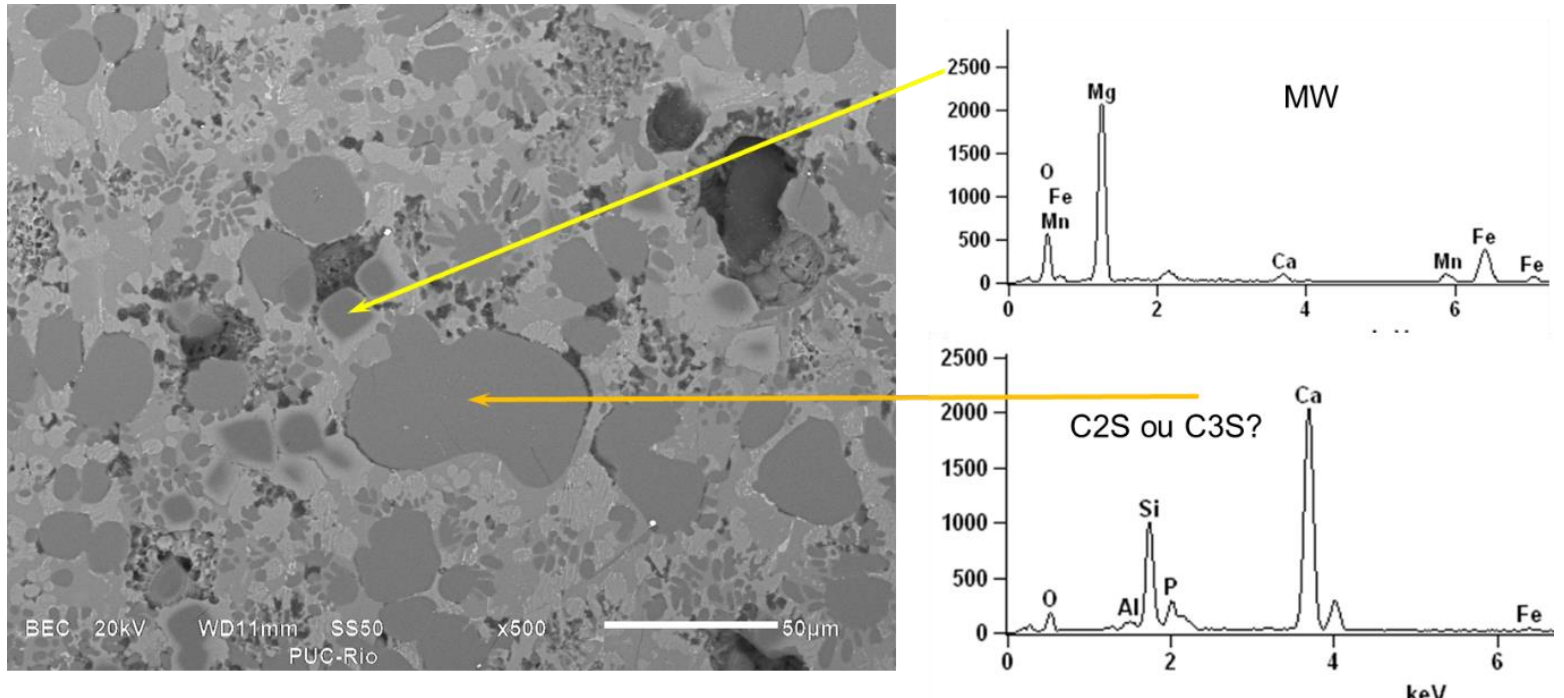

Figura 7. Imagem de elétrons secundários (SE) no MEV de amostra da escória após o tratamento da corrida "214". Uma das partículas de magnesiowustita, (com formato aproximado regular) é indicada assim como a análise por EDS deste ponto. Uma partícula de solução sólida dissilicato ou trissilicato de cálcio (C2S ou C3S) e sua análise por EDS são também indicados (ver texto).

Com a adição posterior de dolomita crua à escória de final do sopro "214", foi realizado o tratamento de slag splashing, com sopro de nitrogênio na ordem de $3000 \mathrm{Nm}^{3}$, logo após a adição da dolomita crua à escória de fim de sopro. O sopro de nitrogênio foi realizado após a adição da dolomita crua e sem a realização de basculamentos do forno para favorecer a calcinação e dissolução do material na escória primária.

A análise da escória pós-tratamento indica diferentes fases presentes, associadas a mudança de composição química da escória. A Tabela 3 apresenta estes resultados, 
com a mudança da composição química da escória "214". Como não foi empregado um padrão para análise no EDS, não foi possível determinar se a segunda fase de saturação, medida por EDS, era C2S ou C3S. É possível que o uso de tempos mais longos de contagem no EDS possa vir a ajudar na eliminação desta dúvida, mas o caminho mais seguro será o uso de padrões na próxima análise, uma vez que o estudo está em execução, ainda. Os cálculos com os dois diferentes bancos de dados indicam que provavelmente a fase observada é C3S. Os resultados de DRX não ajudam a esclarecer este ponto pois os dois silicatos são observados, uma vez que a amostra não solidificou em condições próximas ao equilíbrio.

Tabela 3. Fases em que a escória de tratamento da corrida "214" estaria saturada

\begin{tabular}{|c|c|c|c|}
\hline Método & MEV+EDS & TCOX & SLAG mod. \\
\hline Fases & $\begin{array}{c}\text { MW, (Ca,Fe)O e } \\
\text { C2S OU C3S }\end{array}$ & $\begin{array}{c}\text { MW, (Ca,Fe)O } \\
\text { C3S }\end{array}$ & $\begin{array}{c}\text { MW, (Ca,Fe)O, } \\
\text { C3S }\end{array}$ \\
\hline
\end{tabular}

A Figura 8 apresenta uma análise de EDS em linha em uma escória de após o tratamento, apresentando ao longo de linha uma MW com maior presença de FeO no seu entorno. Uma característica interessante é que em praticamente todas as escórias de splashing/coating, a camada rica em $\mathrm{FeO}$ em torno da MW parece bem mais espessa.

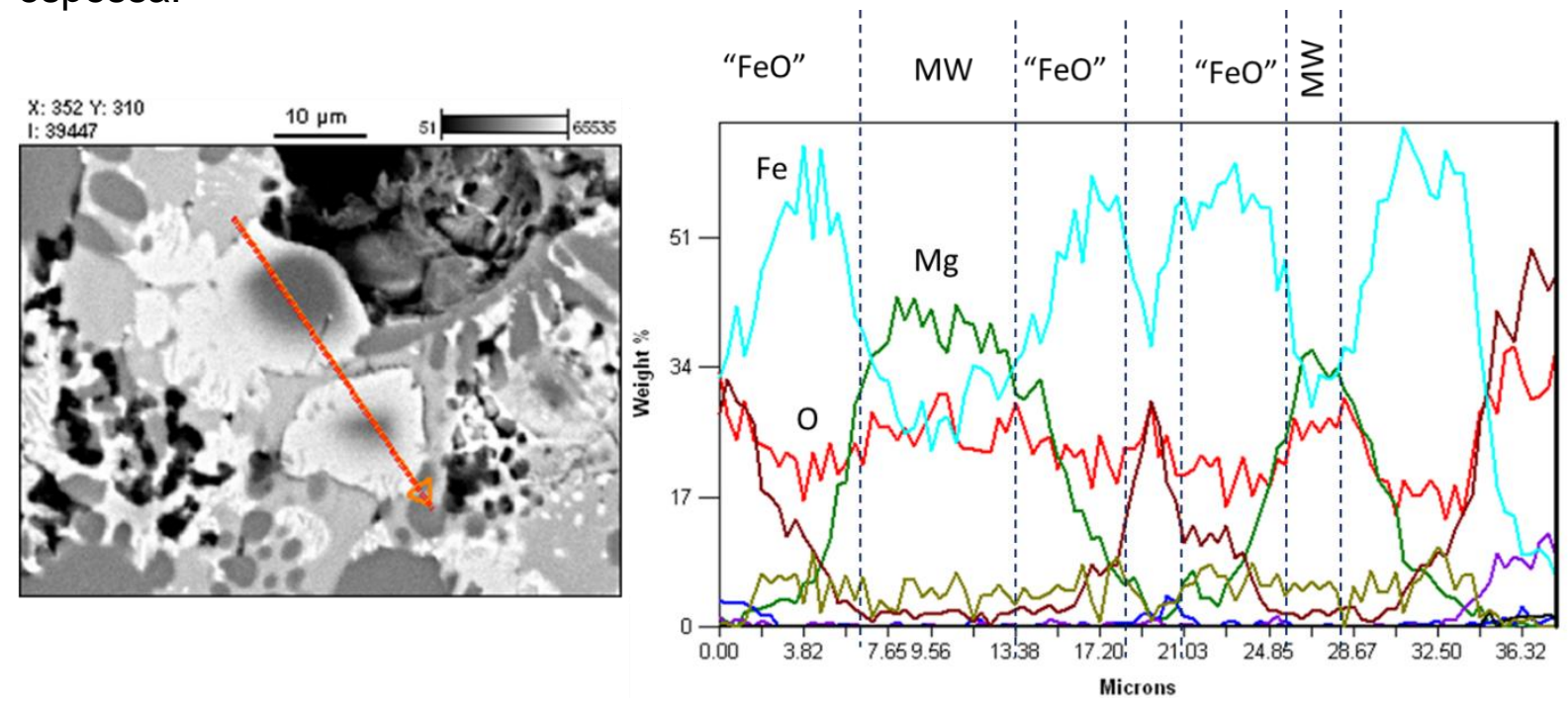

Figura 8. Escória de tratamento da corrida "214". Análise por EDS em linha, ao longo da linha vermelha indicada na foto obtida por MEV. Novamente observa-se uma camada rica em FeO em torno das partículas de geometria regular de MW.

O resultado da análise de EDS da partícula de MW foi comparado com os valores obtidos por DRX e os valores calculados, com base na composição química média da escória, usando os dois bancos de dados. O resultado da comparação é mostrado na Tabela 4.

Tabela 4. Teor de MgO medido e calculado na MW da escória de tratamento da corrida "214"

\begin{tabular}{|c|c|c|c|c|}
\hline Método & DRX Rietveld & EDS & TCOX & SLAG mod. \\
\hline$\% \mathrm{MgO}$ & 85 & 69 & 66 & 66 \\
\hline
\end{tabular}

Embora os valores de $\mathrm{MgO}$ na $\mathrm{MW}$ sejam superiores na análise por $\mathrm{DRX}$, os valores calculados e medidos por EDS são consistentes entre si, e mais baixos. 


\subsection{RESULTADOS DOS CÁLCULOS TERMODINÂMICOS}

As simulações termodinâmicas foram realizadas utilizando dois bancos de dados descritos na seção 2 e são discutidas a seguir

\subsection{1 - Temperatura Liquidus da escória}

A Figura 10 apresenta as temperaturas liquidus calculadas para as escórias em função do seu teor de $\mathrm{MgO}$. Os dois bancos de dados apresentam a tendência correta, para escórias de conversor, do aumento da temperatura liquidus com o teor de $\mathrm{MgO}$. O banco de dados SLAG3 Mod. estiva valores mais elevados do que o banco TCOX6 para esta temperatura. No caso do banco de dados TCOX6 é possível verificar na literatura [13], p.ex. as evidencias do ajuste a temperaturas liquidus medidas. No caso do banco de dados SLAG3 modificado, como discutido acima (Figura 3) é possível que haja, efetivamente, uma estimativa algo exagerada da T liquidus.

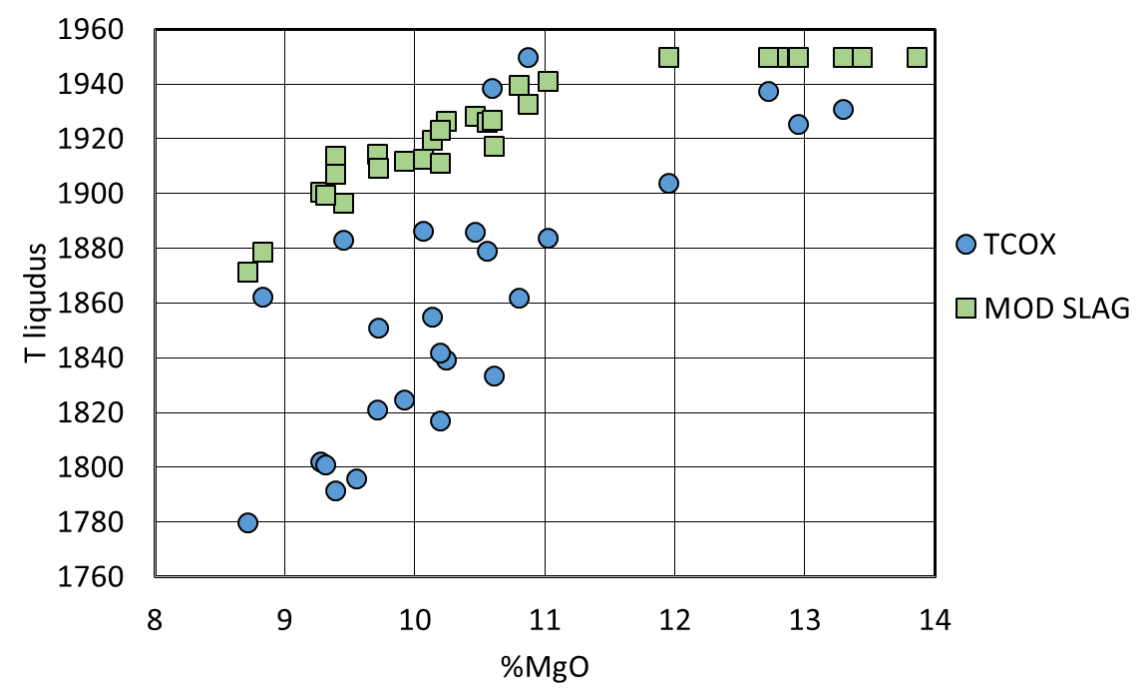

Figura 10. Temperaturas liquidus $\left({ }^{\circ} \mathrm{C}\right)$ estimadas pelos dois modelos em função do teor de $\mathrm{MgO}$ das escórias amostradas.

\subsection{2 - Temperatura Solidus da Escória}

Os cálculos com o banco de dados TCOX6 indicam que o final da solidificação, em equilíbrio, das escórias em questão, se passa em um invariante envolvendo uma calcioferrita, a fase C2F. No banco de dados SLAG3 modificado, criado para o estudo de reações metal-escória, esta fase, por ser uma fase que ocorre a temperaturas relativamente baixas, não foi incluída. Assim, o invariante final da solidificação das escórias, neste banco de dados, envolve o aluminato C3A e ocorre a temperatura significativamente mais elevada do que o previsto pelo TCOX6, como mostra a Figura 11. 


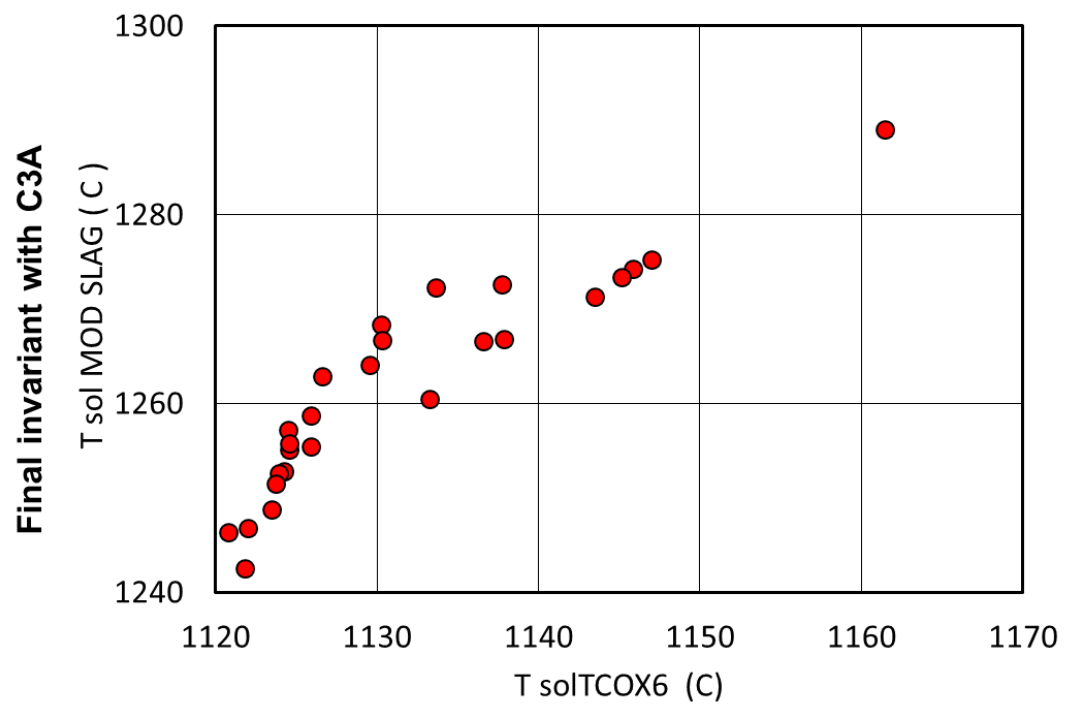

Final invariant with $\mathrm{C} 2 \mathrm{~F}$

Figura 11. Comparação entre as temperaturas solidus calculadas pelos dois modelos termodinâmicos empregados.

\subsection{COMPOSIÇÃO QUÍMICA DA MAGNESIOWUSTITA}

Finalmente, buscou-se verificar a eventual correlação entre os métodos disponíveis para a determinação da composição da magnesiowustita, comparada com as medidas de composição química da escória. Em princípio, é esperada a existência, para escórias similares, de correlações razoáveis entre a composição da escória e a composição da MW. Dentre as variáveis da composição química buscou-se, como variável independente, aquela que apresentou melhor correlação com os valores estimados por DRX. Neste caso, o teor de FeO da escória foi a variável escolhida. $\mathrm{Na}$ Figura 12 são apresentadas, em função do teor de $\mathrm{FeO}$ da escória, o teor de $\mathrm{MgO}$ na magnesiowustita determinado por DRX e pelos cálculos empregando os bancos de dados TCOX6 e SLAG3 mod.

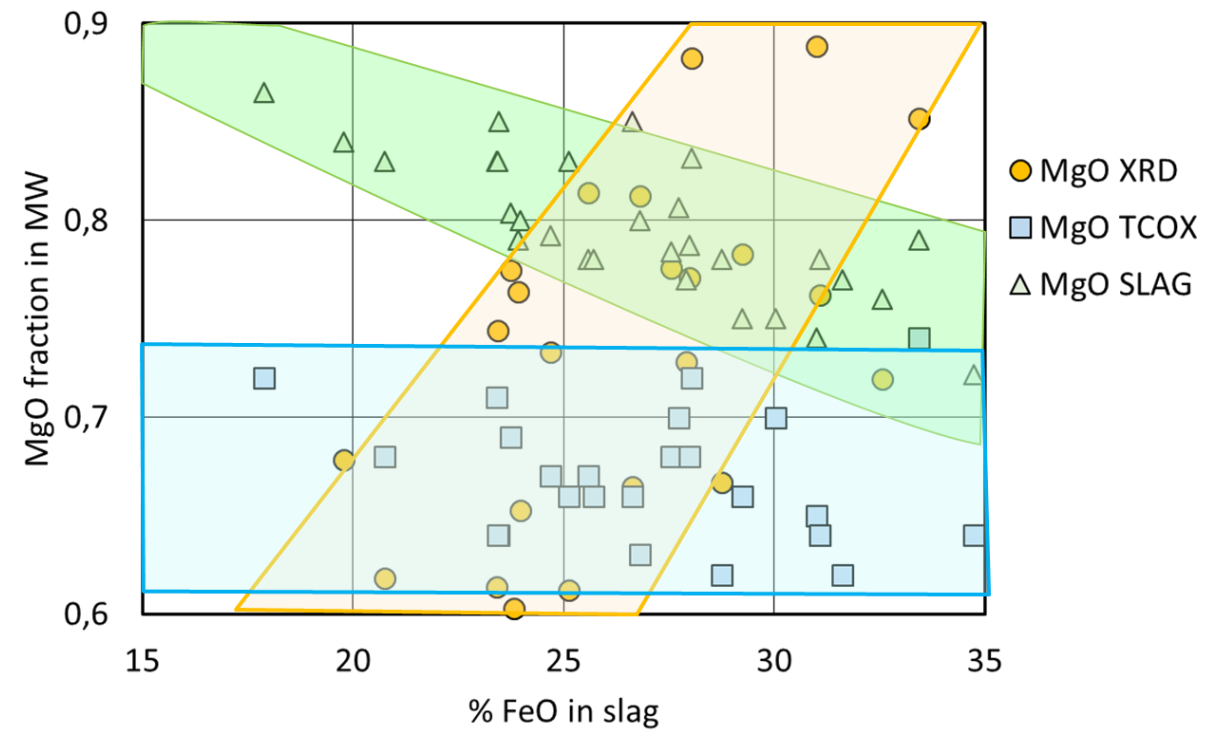

Figura 12. Variação da composição da magnesiowustita com o teor de $\mathrm{FeO}$ na escória para: Medidas experimentais por DRX, cálculos pelos bancos de dados, TCOX6 e SLAG modificado. 
A dispersão entre os valores previstos pelos diversos métodos é claramente observada. Na próxima etapa do estudo espera-se expandir o universo de análises por EDS, com precisão aumentada, e obter amostras que solidifiquem em condições mais próximas ao equilíbrio. Adicionalmente, acredita-se que a compreensão do mecanismo de formação da camada de FeO observada em torno das partículas de MW (Figuras 6 e 8) possam colaborar para a melhor compreensão dos fenômenos envolvidos e das dificuldades associadas a previsão da composição da MW.

\section{CONCLUSÃO}

Em condições operacionais em um conversor a oxigênio de sopro combinado, as adições de $\mathrm{MgO}$ se dissolvem no tempo de corrida formando escórias saturadas em magnesiowustita e não em $\mathrm{MgO}$, como previsto nos diagramas de equilíbrio. Aparentemente a dissolução da dolomita crua e da dolomita calcinada são rápidas, uma vez que $\mathrm{MgO}$ não é observado, mesmo em condições posteriores as adições para proteção do refratário.

Embora discrepâncias tenham sido observadas entre os resultados dos cálculos de termodinâmica computacional empregando diferentes bancos de dados, tais cálculos são de grande utilização na previsão das fases presentes nas etapas de formação de escória e tratamento de splashing/coating.

O mecanismo de dissolução e formação da magnesiowustita em condições industriais deve ser melhor investigado, assim como estudos complementares para a compreensão da formação da camada rica em FeO em torno da MW devem ser realizados. Analises por EDS sem padrão tem pouca precisão para a determinação dos silicatos presentes e estima-se que o uso de padrões internos associados a tempos mais longos de aquisição de dados sejam essências para analises que permitam a compreensão dos silicatos eventualmente presentes na saturação, assim como em um futuro estudo que envolva determinação de compostos contendo fósforo e manganês.

\section{Agradecimentos}

Os autores agradecem a CSN pela permissão para a publicação dos resultados. A Magnesita pela preparação para petrografia das amostras. ACS e RRA agradecem ao CNPq, CAPES e FAPERJ por auxílios em diferentes etapas da pesquisa.

\section{REFERÊNCIAS}

1 Fruehan R, editor. Making, Shaping, and Treating of Steel, Steelmaking And Refining Volume. 11th Edition. Pittsburgh PA: AISE Steel Foundation; 1998.

2 K C Mills, Y Su, A B Fox, Z Li, R P Thackray, H T Tsai. A review of slag splashing. ISIJ International. 2005;45(5):619-33.

3 G Trömel, K. Koch, W. Fix, N Grosskurth. The Influence of Magnesium Oxide on Equilibrium in the System MgO-CaO-FeO-SiO2 and on Sulphur Partition at $1600 \mathrm{C}$. Archiv fur das Eisenhuttenwesen. 1969;40(12):969-78.

4 UMAKOSHI M, MORI K, KAWAI Y. Dissolution rate of burnt dolomite in molten FetOCaO-SiO2 slags. Transactions of the Iron and Steel Institute of Japan. 1984;24(7):532539.

5 Matsushima M, Yadoomaru S, Mori K, Kawai Y. A Fundamental Study on the Dissolution Rate of Solid Lime into Liquid Slag. Transactions ISIJ. 1977;17(Lime Dissolution):442-9. 
6 Schurmann E, Kolm I. Dissolution behavior of dolomite in steelmaking slags, solubility limits and impact on lining wear. Steel Research. 1986;57(2):51-7.

7 Satyoko Y, Lee WE. Dissolution of dolomite and doloma in silicate slag. British Ceramic Transactions. 1999 Jun;98(6):261-5.

8 Andersson JO, Helander T, Höglund L, Shi P, Sundman B. Thermo-Calc \& DICTRA, computational tools for materials science. Calphad. 2002;26(2):273-312.

9 VDEh. Slag Atlas. Verlag Stahleisen, Dusseldorf; 1995.

10 CAMPOS, V. F. Tecnologia de fabricação do aço líquido. Belo Horizonte: UFMG, 1980. Volume 1. Vol. 1, p 77-114;

11 Dogan, N.; Brooks, A, G.; Rhamdhani, A, M. Kinetics of Flux Dissolution in Oxygen Steelmaking. ISIJ International, vol. 49, n. 10, p, 1474 - 1482, june 2009;

12 Hamano, T., Fukagai, S., Tsukihashi, F. Reaction Mechanism between Solid CaO and FeOx-CaO-SiO2-P2O5 Slag at 1573 K. ISIJ International, Vol. 46 (2006), No. 4, pp. 490495.

13 Mao H, Fabrichnaya O, Selleby M, Sundman B. Thermodynamic assessment of the MgOAl2O3-SiO2 system. Journal of Materials Research. 2005 Apr;20(04):975-86. 